\title{
Educación formal e informal en confinamiento: una creciente desigualdad de oportunidades de aprendizaje
}

\author{
Formal and Informal Education During the Lockdown: \\ A Growing Inequality of Learning Opportunities
}

\author{
Xavier Bonal y Sheila González'
}

\section{Resumen}

El cierre de escuelas en Cataluña para contener los efectos de la COVID-19 obligó a los centros educativos y las familias a reaccionar en poco tiempo y sin planificación previa ante un nuevo escenario de enseñanza. Este artículo evalúa el impacto del cierre escolar sobre la desigualdad de aprendizaje entre niños de diferentes contextos sociales en Cataluña. A partir de los datos de una encuesta en línea realizada entre el 26 y el 30 de marzo $(n=35.419)$ a familias con hijos entre 3 y 18 años, nuestro análisis muestra que las oportunidades de aprendizaje varían significativamente en función de las características del alumnado. Durante el confinamiento, algunas escuelas consiguieron adaptarse a las nuevas circunstancias con mejores medios que otras. De igual forma, el capital socioeconómico y cultural de las familias explica que la clase media pudiera mantener estándares más altos de calidad educativa, mientras que aquellas familias socialmente desfavorecidas mostraban una menor exposición a las oportunidades de aprendizaje, tanto en tiempo como en experiencias (tareas escolares y actividades extracurriculares). En la parte final del artículo, destacamos la importancia del papel de la escuela para garantizar oportunidades de aprendizaje para los niños de bajos niveles socioeconómicos y discutimos algunas de las implicaciones políticas de nuestros resultados.

\section{Palabras clave}

Desigualdad educativa, desigualdad acumulativa, brecha digital, cierre escolar, actividades extraescolares, educación informal.

\section{Abstract}

The lockdown of schools in Catalonia to confront the effects of COVID-19 forced schools and families to react very rapidly to a new scenario of teaching and learning processes without previous planning. This paper assesses the impact of the school lockdown on the learning gap between children from different social backgrounds in Catalonia. Based on data from an online survey ( $\mathrm{n}=35.419)$ to families with children aged 3 to 18 years old carried out between 26 and 30 of March, our analysis shows that learning opportunities among children vary significantly between children with different characteristics. During the lockdown, some schools were able to adapt to the new circumstances with better means than others. Likewise, the structure and size of families' forms of capital explain that middle-class families were able to maintain higher standards of education quality, while children from socially disadvantaged families had few learning opportunities both in terms of time and learning experiences (schoolwork and maintenance of after-school activities). In the final part of the paper we highlight the importance of the role of the school to ensure learning opportunities for children from low socioeconomic backgrounds and discuss some policy implications of our findings.

\section{Keywords}

Learning gap, cumulative disadvantage, digital divide, school closure, afterschool activities, informal education.

\section{Cómo citar/Citation}

Bonal, Xavier y González, Sheila (2021). Educación formal e informal en confinamiento: una creciente desigualdad de oportunidades de aprendizaje. Revista de Sociología de la Educación-RASE, 14 (1), 44-62. http://dx.doi.org/10.7203/RASE.14.1.18954.

\footnotetext{
1 Xavier Bonal, Universitat Autònoma de Barcelona, xavier.bonal@uab.cat; Sheila González, Universitat Autònoma de Barcelona, sheila.gonzalez@uab.cat. Globalización, Educación y Politicas Sociales (GEPS)
} 


\section{Introducción}

Para reducir la propagación de la pandemia la mayor parte de gobiernos ha optado por la reducción del contacto físico, hecho que ha conllevado el cierre de los centros escolares. En Cataluña, el cierre de las escuelas se produjo el 13 de marzo de 2020, un día antes de que el gobierno español aprobara el Estado de alarma. Las escuelas y las familias tuvieron que reaccionar rápidamente ante un nuevo escenario de enseñanza y aprendizaje, sin planificación previa y con ausencia de pautas por parte del Ministerio de Educación o de las Consejerías de Educación de las Comunidades Autónomas. En este contexto, algunas escuelas pudieron adaptarse a las nuevas circunstancias con mejores medios que otras. Del mismo modo, las condiciones de confinamiento y el capital de las familias produjeron diferencias significativas en las oportunidades de aprendizaje entre los niños y niñas de diferentes entornos socioeconómicos.

La pandemia de COVID-19 no tiene precedentes en términos de efectos sobre la economía y la vida social, pero no es completamente nuevo para las escuelas experimentar períodos de cierre. Las pandemias anteriores (como el ébola o la gripe), las huelgas de docentes, los desastres naturales o los conflictos violentos han obligado a las escuelas en distintos sitios del planeta a detener sus actividades. Tanto los investigadores como las organizaciones internacionales han estudiado los efectos del cierre escolar sobre el aprendizaje de los estudiantes y han observado reiteradamente una pérdida en la adquisición de habilidades y competencias, pérdida particularmente alta en el caso de niños y niñas de entornos vulnerables. Es muy probable que la evaluación de los efectos de la pandemia actual arroje unos resultados parecidos respecto a su impacto en las competencias de los estudiantes y que aumenten las desigualdades educativas existentes.

En este artículo mostramos cómo el cierre escolar produce oportunidades desiguales de aprendizaje en educación formal y no formal para niños y jóvenes de diferentes entornos socioeconómicos y que asisten a diferentes tipos de escuelas. La exposición al aprendizaje (tanto formal como no formal) entre los niños y niñas de hogares de bajos ingresos es menor que la de sus pares de familias de mayores ingresos, lo que aumenta irremediablemente la brecha de aprendizaje existente entre ellos.

En base a los resultados de una encuesta distribuida durante la segunda semana del estado de alarma, el artículo refleja cómo los antecedentes sociales y las condiciones de confinamiento de los estudiantes están asociados a sus oportunidades de aprendizaje. Estas oportunidades desiguales pueden, a su vez, exacerbar las desigualdades existentes en la adquisición de habilidades y el rendimiento académico.

El documento se estructura del siguiente modo. Primero revisamos la evidencia existente del cierre de los períodos escolares en las pérdidas de aprendizaje y su impacto desigual en diferentes grupos de estudiantes. La siguiente sección proporciona información sobre el contexto en el que se distribuyó la encuesta y la metodología para analizar los datos. Los principales resultados con respecto a las desigualdades en la educación formal y no formal se muestran en la sección siguiente, mientras que la sección final concluye y discute algunas implicaciones políticas de nuestros hallazgos.

\section{Desigualdades de aprendizaje cuando la escuela cierra}

A pesar de lo imprevisto del cierre escolar, la investigación sobre los efectos educativos derivados del mismo ha sido notablemente rica ya desde los primeros meses. Obviamente, la mayoría de los estudios existentes miden los efectos a corto plazo del confinamiento. Ciertamente, es demasiado pronto para saber si estos efectos pueden considerarse una pérdida de aprendizaje o simplemente un efecto temporal 
como resultado de un desuso que se pueda recuperar fácilmente con la práctica (Bonal y González, 2020; Coe et al., 2020). Más allá de la evidencia más reciente, existen varios estudios que tratan los impactos de la ausencia escolar en otros momentos y contextos sobre los resultados del aprendizaje. Estos estudios pueden ayudarnos a comprender los posibles efectos del cierre actual sobre el aprendizaje y los mecanismos por los que se producen las desigualdades educativas. El absentismo, las huelgas docentes, las vacaciones escolares de verano o los conflictos bélicos son algunas de las razones por las cuales las escuelas han reducido o incluso detenido su actividad en el pasado. Esta evidencia identifica la existencia de una pérdida general de aprendizaje, que puede ser más o menos severa dependiendo del desempeño previo de los estudiantes, las características de su familia, edad o trayectoria educativa, entre otros factores.

El cierre escolar, incluso cuando se ofrece educación a distancia, generalmente implica una reducción en el tiempo de instrucción. Los estudios han identificado consistentemente una relación positiva entre el tiempo de aprendizaje y el rendimiento de los estudiantes y otros resultados educativos (Abadzi, 2009; Hanushek, 2015; Scheerens, 2014). Por lo tanto, el tiempo de instrucción se ha utilizado como un proxy de «oportunidades para aprender». Las variables que miden la exposición al currículum parecen tener un mayor efecto en el aprendizaje, incluso más que las variables relacionadas con el comportamiento del maestro o el clima escolar. Se ha identificado una exposición prolongada y repetida a estímulos y feedback del profesorado como un recurso educativo clave. Aunque la evidencia no siempre es concluyente (Cattaneo et al., 2017), la mayoría de los resultados sugieren que los estudiantes de origen migrante y aquellos de entornos socioeconómicos más vulnerables experimentan procesos de aprendizaje más lentos, por lo que pueden beneficiarse de forma más clara de un aumento en el tiempo de exposición al aprendizaje en la escuela (Dahmann, 2015; Gromada y Shewbridge, 2016; Huebener et al., 2017). En definitiva, no asistir a la escuela reduce las oportunidades de aprendizaje para todos, pero lo hace particularmente en el caso de los hijos e hijas de familias menos instruidas o de bajos ingresos.

El absentismo escolar o docente también puede entenderse como una reducción en el tiempo de instrucción. El absentismo comporta un rendimiento académico más pobre y afecta al desarrollo de habilidades, competencias y comportamientos adecuados para el éxito educativo, a la vez que aumenta la probabilidad de abandono escolar. Sus efectos van más allá del ambiente escolar y pueden generar comportamientos de riesgo como la delincuencia juvenil, el alcoholismo, el uso de drogas, las prácticas sexuales de riesgo o los embarazos no deseados, así como otros efectos en la edad adulta (desempleo, alcoholismo) que disminuyen las oportunidades de vida para los jóvenes absentistas (Abadzi, 2009; Coe et al., 2020; González Motos, 2020).

En Cataluña, como en el resto de España y en otros países europeos, el cierre escolar entre marzo y junio de 2020 ha enlazado con las vacaciones de verano, hecho que ha supuesto seis meses sin clases presenciales. Los estudios que comparan el progreso del aprendizaje durante el año escolar y las vacaciones de verano han demostrado consistentemente que el rendimiento se ralentiza o disminuye durante las vacaciones estivales. Aunque no hay acuerdo sobre la magnitud de la pérdida de aprendizaje durante las vacaciones (von Hippel, 2019), los estudios revelan que la pérdida es mayor en matemáticas que en lectura y especialmente acentuada en el caso de los estudiantes de grado superior (Kuhfeld y Tarasawa, 2020b, 2020a). Además, las investigaciones señalan que esta pérdida no afecta a todos los estudiantes por igual, por lo que las desigualdades educativas aumentan entre los niños y niñas de entornos socialmente favorecidos y desfavorecidos durante los períodos de verano (Alegre, 2016). 
Más allá de la evidencia proveniente de alteraciones en la organización del año escolar ordinario (absentismo, vacaciones de verano, tiempo de instrucción), otras investigaciones han analizado los efectos causados por factores exógenos. La literatura sobre el cierre de colegios por causas climatológicas (nevadas, huracanes) contribuye a una mejor comprensión de las posibles consecuencias de un cese escolar abrupto. La mayor parte de la evidencia se basa en muestras pequeñas, es geográficamente específica y se refiere a cortos períodos de descanso. Sin embargo, en todos los casos los estudios encuentran un efecto claro de cada día de cancelación de la escuela sobre el rendimiento, especialmente entre los estudiantes que asisten a escuelas pobres o aquellos que provienen de familias de bajos ingresos (Goodman, 2014; Kuhfeld y Tarasawa, 2020a; Marcotte y Hemelt, 2008).

Las huelgas docentes también han forzado una cancelación repentina de clases. Las huelgas de larga duración afectan a los resultados de los estudiantes (rendimiento y repetición de grado, principalmente) al reducir el tiempo que los niños y niñas asisten a las escuelas, pero también afectan a la calidad de la escuela. Estas investigaciones también han hallado efectos heterogéneos, siendo los niños de las familias más vulnerables los más afectados por las interrupciones escolares (Jaume y Willén, 2019).

La principal diferencia entre el parón escolar examinado por estudios previos y el cierre actual es que ahora las escuelas han mantenido cierto contacto con los estudiantes, principalmente a través de la instrucción en línea. No obstante, a pesar de los esfuerzos para apoyar el aprendizaje remoto, muchos estudiantes no han tenido acceso al mismo. Los datos existentes sobre la brecha digital y las encuestas desarrolladas durante el confinamiento indican que los y las docentes no han podido contactar con un número significativo de estudiantes, principalmente debido a la falta de conexión a Internet o de dispositivos adecuados para seguir el aprendizaje a distancia (Kuhfeld y Tarasawa, 2020a; Van Lancker y Parolin, 2020). Para el caso español, los datos coinciden en identificar al menos un $10 \%$ de los hogares con menores sin disponibilidad de ordenador y/o sin conexión a internet, observándose importantes diferencias en función de la renta, nivel de estudios y lugar de residencia (Cabrera, 2020).

Aun cuando los alumnos han podido conectarse, el aprendizaje remoto parece haber ampliado la brecha de rendimiento existente entre los estudiantes de entornos socioeconómicos desiguales (Coe et al., 2020; Rogero-García, 2020). Una investigación reciente en Brown y Harvard sobre el uso de un programa de matemáticas en línea (Zearn) antes y durante el confinamiento muestra una fuerte disminución del progreso de los estudiantes en matemáticas en las aulas ubicadas en códigos postales de bajos ingresos, mientras que en los códigos postales de altos ingresos no se observan cambios (Goldstein, 2020). Antes de la pandemia, el aprendizaje presencial ya había demostrado ser más efectivo que el aprendizaje a distancia. Los resultados de los estudiantes a través de la enseñanza en línea son, en promedio, más pobres que los de la instrucción presencial. Las condiciones para un aprendizaje remoto efectivo (buena conexión, explicaciones claras, sólida ordenación de contenidos y retroalimentación comprensible) no son fáciles de lograr: la brecha digital, pero también la falta de experiencia entre los docentes para proporcionar un aprendizaje a distancia de alta calidad, dificultan las oportunidades de aprendizaje de los alumnos (Kay, 2020; Rogero-García, 2020). Por todo esto, existe el riesgo de que los estudiantes vulnerables tengan un menor acceso a una enseñanza de calidad, lo que amplía la desigualdad (Coe et al., 2020; Kay, 2020; Rogero-García, 2020). Si en condiciones normales se ha demostrado que los estudiantes de familias de bajos ingresos experimentan más interrupciones y disrupciones en el aula (Abadzi, 2009; Alegre y Benito, 2012), un deficiente aprendizaje a distancia puede entenderse como un nuevo obstáculo para la efectividad del aprendizaje. 
Además de la organización escolar o de las estrategias de aprendizaje a distancia desarrolladas por los docentes, la participación de las familias en el aprendizaje de los niños y niñas se vuelve más importante cuando la escuela es reemplazada por la instrucción en el hogar. Diversos estudios han demostrado que la participación de las familias y la calidad del aprendizaje en el hogar mejoran los resultados académicos. La investigación en este campo ha observado consistentemente un mayor logro académico entre los estudiantes cuyas familias participan activamente en el proceso educativo, y una relación clara entre la participación en los procesos de aprendizaje y el origen de las familias en términos de clase social o procedencia. Se han documentado ampliamente las capacidades desiguales para ayudar a los niños y niñas con sus tareas y los diferentes usos del tiempo familiar (Cabrera, 2020, Meyer et al., 2017; Mora \& Escardíbul, 2018; Oreopoulos et al, 2006, Pérez et al., 2013). Por lo tanto, es probable que las reacciones familiares al cierre escolar produzcan diferencias significativas en las oportunidades de aprendizaje de niños de diferentes entornos sociales (Burgess y Sievertsen, 2020).

La pandemia ha supuesto la desaparición de la escuela presencial y ha mostrado, sin atenuantes, la desventaja del alumnado más vulnerable, aquel que se encuentra más alejado de la institución escolar y menos expuesto a dinámicas educativas parecidas a las que acontecen en los contextos escolares. Pero, además, como señala Tarabini (2020), no solo se ha observado la existencia de amplias brechas entre familias para ejercer su función educativa, sino también la amplia distancia entre los propios centros educativos para desarrollar su papel igualador.

Independientemente de sus causas y mecanismos, las crisis anteriores tuvieron un efecto más intenso y negativo sobre los resultados del aprendizaje en aquellos contextos con mayores proporciones de familias desfavorecidas (Borse et al., 2011; Chen et al., 2011; Iqbal et al., 2020; Sadique et al., 2008; Shores \& Steinberg, 2018). Investigaciones previas también sugieren que la pérdida de aprendizaje no solo puede tener un efecto limitado a corto plazo, sino que puede resultar en pérdidas acumulativas (Abadzi, 2009). Del mismo modo, otros analistas sugieren que el cierre de la escuela ampliará la brecha de aprendizaje entre los niños vulnerables y sus compañeros de entornos más acomodados, llegando incluso a revertir el progreso realizado durante la última década para reducir esta brecha (Coe et al., 2020; Van Lancker \& Parolin, 2020).

\section{Contexto, datos y métodos}

El 13 de marzo, el gobierno catalán ordenó el cierre de todas las escuelas y colegios debido a la pandemia de coronavirus. Se obligó a más de un millón de estudiantes de educación obligatoria a quedarse en casa. Durante las dos primeras semanas de confinamiento, el Departamento de Educación de la Generalitat de Cataluña dio instrucciones a las escuelas para no avanzar en contenidos curriculares, en un intento por contener los efectos adversos de la brecha digital sobre las desigualdades educativas. A pesar de ello, algunas escuelas reaccionaron rápidamente a las nuevas circunstancias y comenzaron a aplicar estrategias de aprendizaje a distancia. Otras escuelas detuvieron completamente sus actividades. Entre ambos extremos, hubo un amplio abanico de reacciones y respuestas.

Entre el 26 y el 30 de marzo, después de dos semanas de cierre escolar y antes de que el Gobierno español ordenara un cierre más restringido, lanzamos una encuesta en línea a familias con hijos e hijas entre 3 y 18 años. La encuesta se dirigió a familias con menores matriculados en educación preescolar (de 3 a 5 años), escuela primaria (6 a 12 años), educación secundaria obligatoria (12 a 16 años), y bachillerato y formación profesional de grado medio (16-18 años). 
La encuesta incluyó 78 ítems y un número variable de sub-preguntas filtradas para adaptar el cuestionario al contexto de la persona encuestada. Se incluyeron dos conjuntos de preguntas. En primer lugar, un grupo de cuestiones con el objetivo de caracterizar las diferentes condiciones sociales, espaciales y tecnológicas en las que los menores y sus familias tuvieron que responder al cierre de las escuelas (como el tamaño de la vivienda, la disposición de espacios al aire libre, el acceso a internet o la disponibilidad de dispositivos digitales). En segundo lugar, preguntas para evaluar las oportunidades de aprendizaje de los niños y niñas en educación formal (contacto con su profesorado, frecuencia y tipos de tareas escolares, retroalimentación proporcionada por las escuelas), educación informal (usos del tiempo, actividades familiares, apoyo para llevar a cabo tareas escolares) y prácticas educativas no formales desde el hogar (participación en actividades extraescolares, tipos de actividades y formas de participación). Además, el cuestionario incluía variables para caracterizar las condiciones sociales y económicas de las familias encuestadas, como la estructura familiar, el nivel educativo y el origen étnico de padres y madres y su situación económica y laboral.

Se realizó una breve encuesta piloto para evaluar el cuestionario. Debido al momento excepcional, esta prueba se realizó a través de una estrategia informal: se pidió a diez familias con niños de diferentes edades e itinerarios escolares que respondieran el cuestionario. La versión final del cuestionario se distribuyó a través de varios canales de comunicación (Twitter, Facebook y WhatsApp). Esta estrategia nos ayudó a llegar a diferentes grupos sociales, así como a reducir el sesgo de muestreo no probabilístico relacionado con el uso diferente de las redes sociales (Blank, 2017). También movilizamos contactos estratégicos en áreas residenciales más desfavorecidas para llegar a aquellas familias con menos probabilidades de responder cuestionarios en línea.

79668 personas accedieron al enlace de la encuesta, aproximadamente 58000 comenzaron a responderla y más de 40000 familias la completaron. Después de la exclusión de algunas encuestas debido a diferentes razones (respuestas incompletas, edades de los hijos e hijas fuera de rango, familias no residentes en Cataluña), la muestra final incluyó información de 35419 familias y 59167 menores entre 3 y 18 años. Se trata de una muestra no probabilística y, como se esperaba, las familias de altos ingresos y estudios elevados estaban sobrerrepresentadas. Debido al confinamiento y a la necesidad urgente de recopilación de datos, no pudimos complementar la encuesta en línea con otras estrategias, como llamadas telefónicas o encuesta presencial. El elevado número de respuestas, sin embargo, permitió realizar una «ponderación de no respuesta» para acercar la composición de la muestra a la distribución de la población. Ponderamos la muestra por el nivel de estudios, como variable clave de diferenciación social y cultural, y como una fuente confiable y precisa a través de datos censales. La Tabla I recoge la muestra ponderada y no ponderada.

Tabla I. Muestra ponderada y no ponderada

\begin{tabular}{lccccc} 
& MUESTRA (N) & MUESTRA (\%) & POBLACIÓN (\%) & $\begin{array}{c}\text { PONDERACIÓN } \\
\text { (FACTOR) }\end{array}$ & $\begin{array}{c}\text { MUESTRA } \\
\text { PONDERADA (N) }\end{array}$ \\
\hline Educación obligatoria & 2387 & 6,74 & 27,4 & 4,1 & 9916 \\
\hline Educación postobligatoria & 9911 & 27,98 & 33,0 & 1,2 & 11689 \\
\hline Educación superior & 23121 & 65,28 & 39,6 & 0,6 & 13813 \\
\hline
\end{tabular}


El 86\% de los encuestados eran mujeres, el 89,3\% vivía en hogares biparentales heterosexuales, el 9,6\% en hogares monoparentales y el 1,1\% pertenecía a parejas homosexuales. La mayoría de los hogares estaban compuestos por 4 miembros (53\%), los hogares de 3 miembros representan el $27 \%$ de la muestra y el $16 \%$ de los hogares tenían 5 o más miembros.

La distribución de la muestra según las variables escolares refleja con precisión la distribución entre los sectores escolares públicos y privados. En Cataluña, la proporción de niños en educación obligatoria que asisten a escuelas públicas es del 68\% (69,9\% en nuestra muestra), mientras que el 29\% de los estudiantes asisten a escuelas privadas concertadas $(27,5 \%$ en la muestra) y solo el $2 \%$ están matriculados en escuelas privadas no concertadas (2,6\% en la muestra). La muestra también está bien equilibrada con respecto a las edades de los niños y niñas, con una ligera sobre representación de los más pequeños.

El análisis de datos se organizó dividiendo la información en tres bases de datos diferentes de acuerdo con diferentes unidades de análisis. Primero, una «base de datos de hogares» incluye información sobre todos los miembros que conviven durante el confinamiento. En segundo lugar, una «base de datos de estudiantes» recopila información sobre todas las actividades de aprendizaje de niños y niñas entre 3 y 18 años. Finalmente, la «base de datos de actividades extraescolares» contiene información sobre cada actividad extracurricular realizada antes y durante el confinamiento. Además, algunas submuestras fueron construidas para llevar a cabo análisis específicos. En este artículo, presentamos algunos datos basados en submuestras por edad o nivel educativo de los estudiantes.

\section{Ampliación de la desigualdad social: de las condiciones de aprendizaje a las oportunidades de aprendizaje}

En esta sección reflejamos los datos sobre las diferencias sociales en las condiciones de aprendizaje durante el confinamiento y su impacto sobre las oportunidades de aprendizaje. Estas oportunidades están condicionadas por las desigualdades en el capital económico y cultural pero también por las respuestas de los centros escolares, el acceso a dispositivos digitales y las posibilidades de apoyo por parte de las familias al aprendizaje. En esta sección revisamos cómo estos factores son impulsores de desigualdades en la educación formal, no formal e informal.

\section{1. Condiciones de aprendizaje en el hogar: diferencias sociales y tecnológicas}

Las condiciones de aprendizaje en el hogar difieren en función de diferentes variables. El espacio físico o el acceso a dispositivos tecnológicos tienen un impacto en las condiciones de aprendizaje.

Nuestra encuesta incluyó preguntas sobre el tamaño del hogar y los espacios exteriores (balcones, terrazas) disponibles, como indicadores de las condiciones espaciales de confinamiento. La mayoría de los hogares disponían de un balcón u otros espacios al aire libre. Sin embargo, existen diferencias significativas entre los residentes según el tamaño del municipio (las viviendas en ciudades tienen menos espacios exteriores) y otros indicadores sociales, como el nivel educativo de los progenitores. Por ejemplo, el 13\% de las familias con un adulto con educación obligatoria no tienen espacios al aire libre, mientras que este es solo el caso del $4 \%$ de los hogares en los que al menos un adulto tiene un título universitario. Asimismo, el $45 \%$ de los hogares más pobres tenían menos de 80 metros cuadrados, un porcentaje que se reduce al 14\% en el caso del quintil de ingresos más rico. 
Las condiciones de conectividad y las oportunidades para acceder a dispositivos tecnológicos son también desiguales, lo que implica diferencias en las capacidades para llevar a cabo las tareas escolares. Como solo pudimos implementar nuestra encuesta en línea, no podemos medir la extensión de la brecha digital real. Los datos del Departamento de Educación del gobierno catalán estiman que había aproximadamente 55000 niños en edad escolar sin conexión a internet (Vallespín, 2020), lo que situaría la brecha digital entre el 10\% y el 15\% de todos los estudiantes en el sistema educativo. Nuestra encuesta revela que el 3,5\% de las familias tenía solo una conexión móvil o ninguna conexión. La mayoría de las familias tenía, en cambio, acceso a una conexión de alta velocidad. Sin embargo, existen diferencias importantes con respecto a las «posibilidades de uso» de la tecnología. Por ejemplo, el 15,3\% de los encuestados declararon que tenían acceso a un solo dispositivo en casa o que solo tenían acceso al teléfono móvil. Calculamos la cantidad de dispositivos por persona y descubrimos que en el $56 \%$ de los casos hay menos de un dispositivo por persona en el hogar. La brecha digital también se puede expresar a través del acceso a dispositivos basados en la composición del hogar. Así, en el 12\% de los hogares con 2 menores solo hay un dispositivo disponible; en los hogares de 3 niños o niñas, el $32 \%$ tiene 2 dispositivos o menos; y en los hogares de 3 niños, el $48 \%$ tiene menos de 3 dispositivos. Así pues, en la mayor parte de familias, la falta de dispositivos reduce sus posibilidades de uso e implica la necesidad de compartir.

El acceso a la tecnología depende del nivel de ingresos de la familia. Teniendo en cuenta el tamaño del hogar, solo el 29\% de las familias más pobres (Q1) tiene acceso a un dispositivo por persona. Entre las familias en Q5, el número de hogares con al menos un dispositivo por persona alcanza el 63\%. El gráfico 1 muestra la proporción de hogares para cada quintil de ingresos que tienen acceso a uno o más dispositivos por persona. Las diferencias se ordenan por ingresos, con un factor de desigualdad de 2,5 entre Q5 y Q1.

\section{Gráfico 1. Hogares con al menos un dispositivo digital por persona, según quintiles de renta}

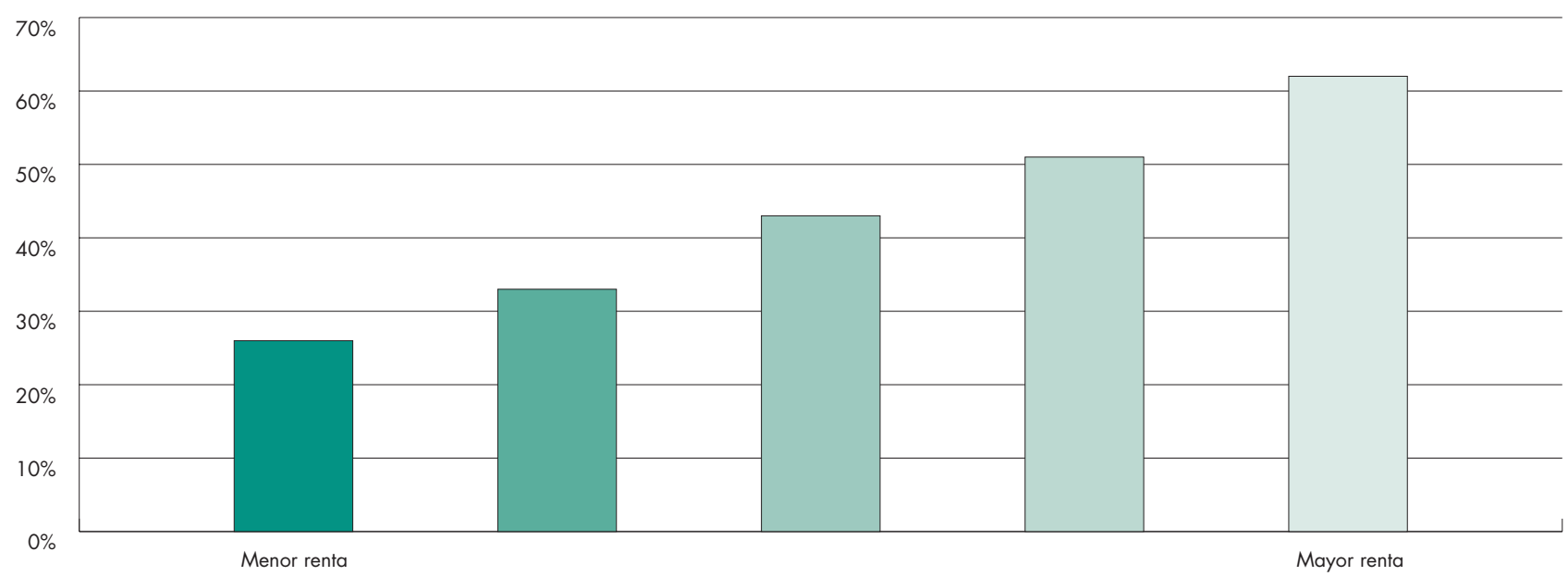

Además, el impacto desigual de la crisis en la situación laboral de los progenitores también altera las condiciones sociales y psicológicas para garantizar un proceso de aprendizaje adecuado. Nuestra 
encuesta reveló que el 13\% de los adultos estaban desempleados antes del cierre y el $80 \%$ trabajaban a tiempo completo. Cuando preguntamos por el impacto de la crisis de la COVID-19 observamos que la situación laboral había cambiado drásticamente: el 23\% de los encuestados indicaron que habían perdido su trabajo y de los que trabajan, solo el 21,5\% iba a trabajar «normalmente». El resto trabajaba desde casa, con el mismo horario o con un horario más flexible (la mayoría, 39\%). Un 17\% de los que trabajaban al inicio de la pandemia consideraban probable perder su trabajo. De los que estaban desempleados en el momento de la encuesta, el 50\% tenía derecho a beneficios de desempleo, mientras que el otro $50 \%$ no lo tenía o desconocía si tenía derecho a tenerlo.

\subsection{Tareas escolares y oportunidades de aprendizaje}

Nuestra encuesta incluyó preguntas sobre cuánto tiempo invertían niños y niñas en las tareas escolar cada día, qué tan frecuente era el contacto con la escuela y sus profesores, con qué frecuencia recibían clases en línea, si los niños recibían tareas para realizar de forma autónoma y con qué frecuencia estas tareas eran revisadas y devueltas a los estudiantes. Teniendo en cuenta la intensidad de todas estas cuestiones, pudimos componer un Índice de Oportunidades de Aprendizaje (IOA). Los indicadores se normalizaron y las frecuencias se agregaron para componer el índice, que oscila entre 0 y 100. Este índice solo se ha aplicado para niños entre 10 y 18 años, ya que las tareas escolares o la enseñanza en línea se mostraron más improbables para edades más tempranas.

El 28,3\% de los estudiantes obtuvo un índice igual a 0 , lo que significa que dedicaban menos de una hora al día a las tareas escolares, casi no tenían comunicación con sus profesores y no recibían tareas para hacer ni eran revisadas. En el otro extremo, el 7,7\% de los estudiantes obtuvo un IOA máximo de 100, lo que significa que dedicaban más de cuatro horas al día al trabajo escolar, tenían contacto frecuente con sus profesores y profesoras y recibían comentarios frecuentes sobre sus tareas escolares. El 80,2\% de todos los estudiantes de la muestra obtuvo un IOA por debajo de 60 puntos.

El puntaje IOA se relaciona positivamente con una serie de variables como tener acceso a un mayor número de dispositivos digitales, estar inscrito en cursos más avanzados (los estudiantes mayores tenían un IOA más alto), ser autóctono y tener mayores ingresos. El alumnado de escuelas concertadas y privadas tuvo puntajes IOA significativamente más altos que el de escuelas públicas. Hay razones que explican esta diferencia. El Departamento de Educación del gobierno catalán anunció que las dos primeras semanas de cierre escolar serían no lectivas. Un número significativo de escuelas públicas no desarrollaron tareas escolares durante este período, a la espera de nuevas instrucciones del Departamento de Educación. A pesar de este anuncio, las escuelas concertadas y privadas no detuvieron su actividad lectiva. Una de las explicaciones plausibles de esta diferencia radica en la dependencia económica de las aportaciones familiares de estas escuelas y a la necesidad de seguir prestando servicio a los usuarios a pesar de las circunstancias excepcionales.

El gráfico 2 muestra cómo el índice IOA se distribuye de manera diferente entre los sectores escolares. La figura revela fuertes diferencias por sector de titularidad para los estudiantes matriculados en los últimos años de educación primaria, secundaria obligatoria y postobligatoria. El gráfico también muestra las diferencias del índice IOA en función de la renta familiar, siendo más bajo entre el alumnado de Q1 que el de Q5, y siendo estas diferencias más acusadas (aunque con un puntaje 
siempre superior) en los centros privados y concertados. Las diferencias entre centros escolares son claras y más intensas que las observadas entre grupos sociales.

\section{Gráfico 2. Oportunidades de Aprendizaje (índice IOA) del alumnado 10 a 18 años, según titularidad de escuela e ingresos familiares (Q1 menor renta, Q5 mayor renta)}

\begin{tabular}{l|l|l|l|l|l|l|l} 
Q1 & Q2 & Q3 & Q4 & Q5
\end{tabular}

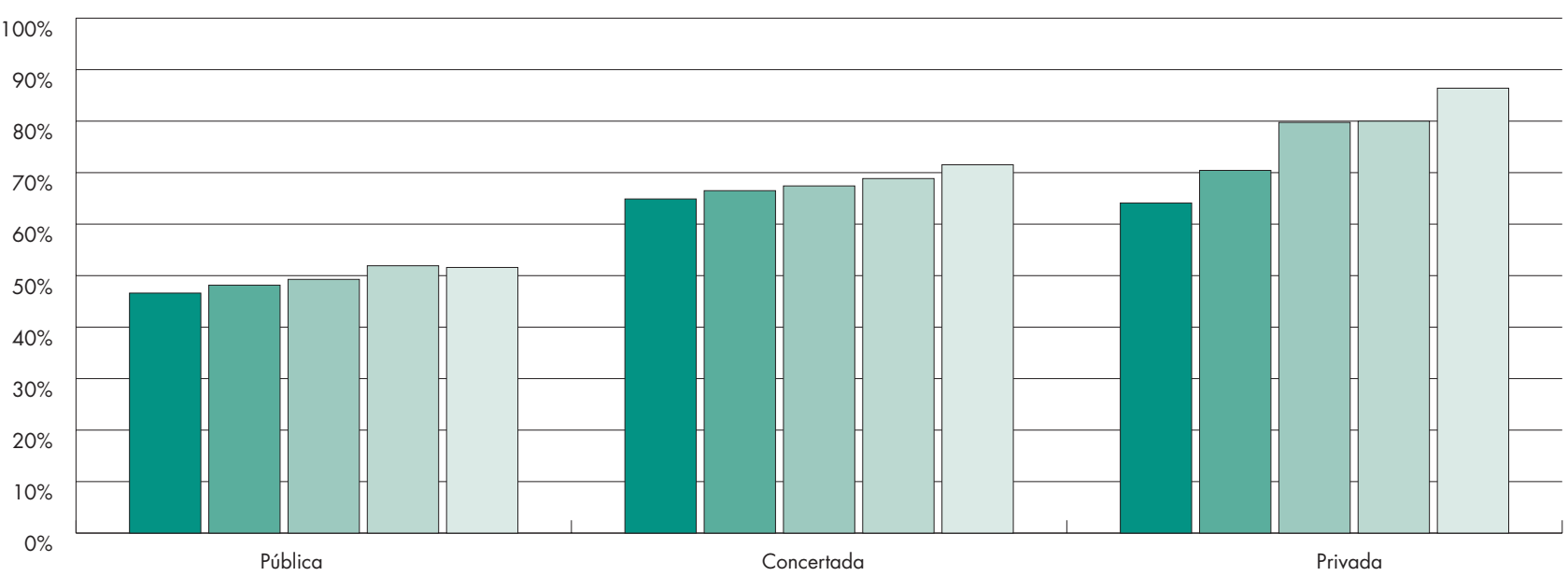

\subsection{El papel de las familias en el proceso de aprendizaje}

La ausencia de escuela aumenta la importancia de las familias como sustitutos de los docentes en el proceso de aprendizaje. Nuestra encuesta incluía preguntas sobre si los adultos de la familia ayudaban a los estudiantes en sus tareas escolares. Un primer resultado muestra importantes diferencias en términos de género: el 79\% de las madres declararon que apoyaban a sus hijos en las tareas escolares, mientras que solo el $43 \%$ de los hombres adultos lo hacía.

Como se esperaba, el apoyo familiar es mayor en el caso de los niños más pequeños. El gráfico 3 muestra que en la educación primaria el apoyo de las madres con educación obligatoria es bastante comparable al de las madres con mayor capital instructivo. Sin embargo, en la educación secundaria obligatoria las diferencias aumentan dramáticamente: solo el 35\% de las madres con educación obligatoria ayudan con la tarea, una cifra que aumenta al $48 \%$ en el caso de las madres más educadas. El apoyo a las tareas escolares en la educación postobligatoria disminuye a porcentajes cercanos al $20 \%$ para todos los grupos, aunque se observan ligeras diferencias entre clases sociales. 


\section{Gráfico 3. Apoyo a las tareas escolares, según ciclo educativo del alumnado y género y nivel instructivo de los progenitores \\ Madre estudios universitarios _ Padre estudios universitarios \\ Madre estudios obligatorios $\quad$ Padre estudios obligatorios}

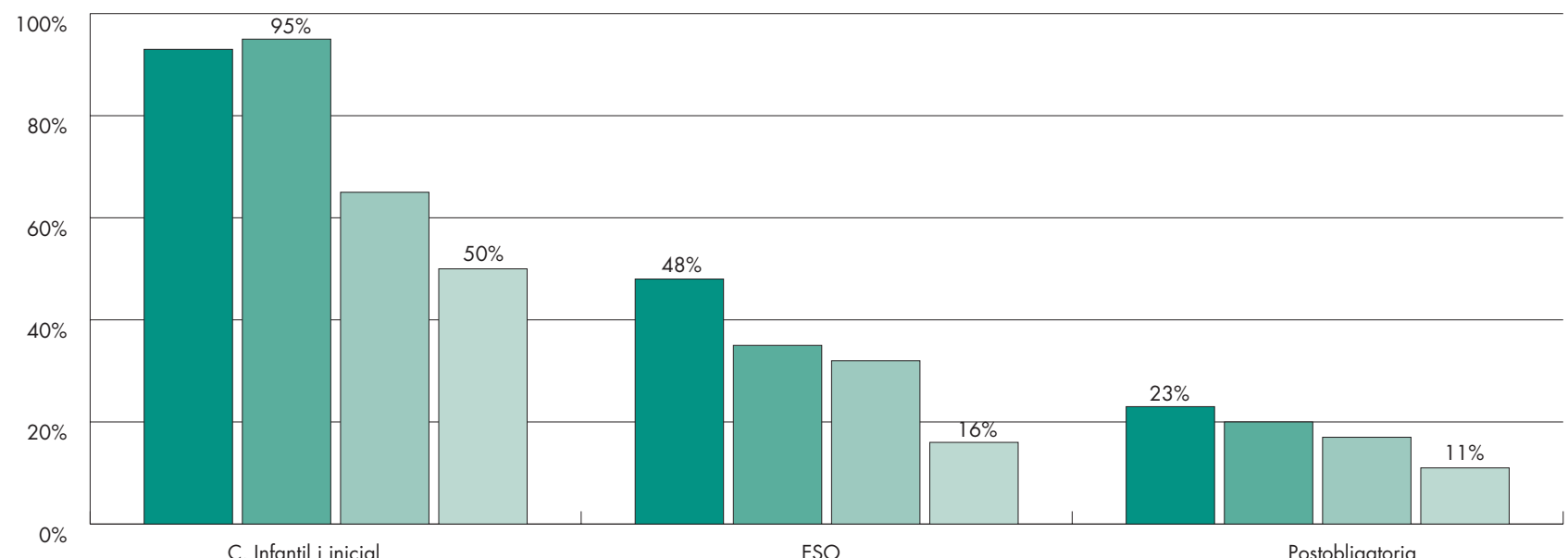

Las razones por las que no se colabora en las tareas escolares son notablemente diferentes según el nivel educativo de las familias. En aquellos hogares con hijos e hijas en educación secundaria obligatoria, el $92 \%$ de las familias con un título universitario que no ayudan a las tareas escolares argumentan que el niño no lo necesita. Esta razón solo es planteada por el $69 \%$ de los encuestados de aquellos hogares con educación obligatoria. En este caso, la falta de conocimiento se argumenta como una razón importante para no brindar apoyo (28\%), una proporción que se reduce al $2 \%$ para aquellos progenitores con un título universitario. Se trata, pues, de una muestra más del reflejo de las diferencias de capital cultural en la capacidad y las posibilidades de las familias para ayudar a los niños y niñas en sus tareas escolares.

Además, el capital cultural familiar y las prácticas cotidianas informales tienen efectos sobre las experiencias y oportunidades de aprendizaje de sus hijos e hijas. Curiosamente, el confinamiento permite evaluar estas actividades de aprendizaje informal bajo condiciones ideales, puesto que se maximiza el tiempo de convivencia e interacción entre los miembros de la familia. El gráfico 4 revela que, en aquellos hogares con niños entre 3 y 8 años, hay tres actividades en las que las diferencias sociales se acentúan especialmente. Primero, acompañar a los niños en lectura es mucho más frecuente en familias con un alto capital cultural: el 59\% de las familias con estudios universitarios responden que los niños de entre 3 y 8 años leen con un adulto todos los días. Esta proporción es de solo el $37 \%$ en el caso de las familias con educación obligatoria. En segundo lugar, las diferencias también son visibles en las prácticas informales de lenguas extranjeras. Un total de $44 \%$ de las familias con estudios universitarios responde que la práctica de lenguas extranjeras se realiza diariamente o varios días a la semana, mientras que esta práctica la lleva a cabo el 36\% de las familias con educación obligatoria. Tercero, el $81 \%$ de las familias con títulos universitarios practican actividades deportivas varias veces a la semana, cifra que se sitúa en el $66 \%$ entre las familias con estudios obligatorios.

En contraste, otras actividades son más frecuentes entre las familias con menor capital cultural. La más relevante es jugar a videojuegos. El 37\% de las familias con educación obligatoria afirman que sus hijos e hijas juegan a videojuegos todos los días o varias veces a la semana. Esta proporción 
es solo del 21,5\% en el caso de madres con estudios universitarios. Las diferencias también son muy significativas en la frecuencia de ver programas educativos de televisión (el 52\% de las madres con educación obligatoria con niños menores de 10 años afirman verlos todos los días frente al 30\% de las madres con estudios universitarios), y en el seguimiento y la realización de actividades educativas disponibles en las redes sociales.

\section{Gráfico 4. Prácticas informales entre el alumnado de 3 a 8 años según nivel instructivo de la madre}

Estudios obligatorios

Estudios universitarios

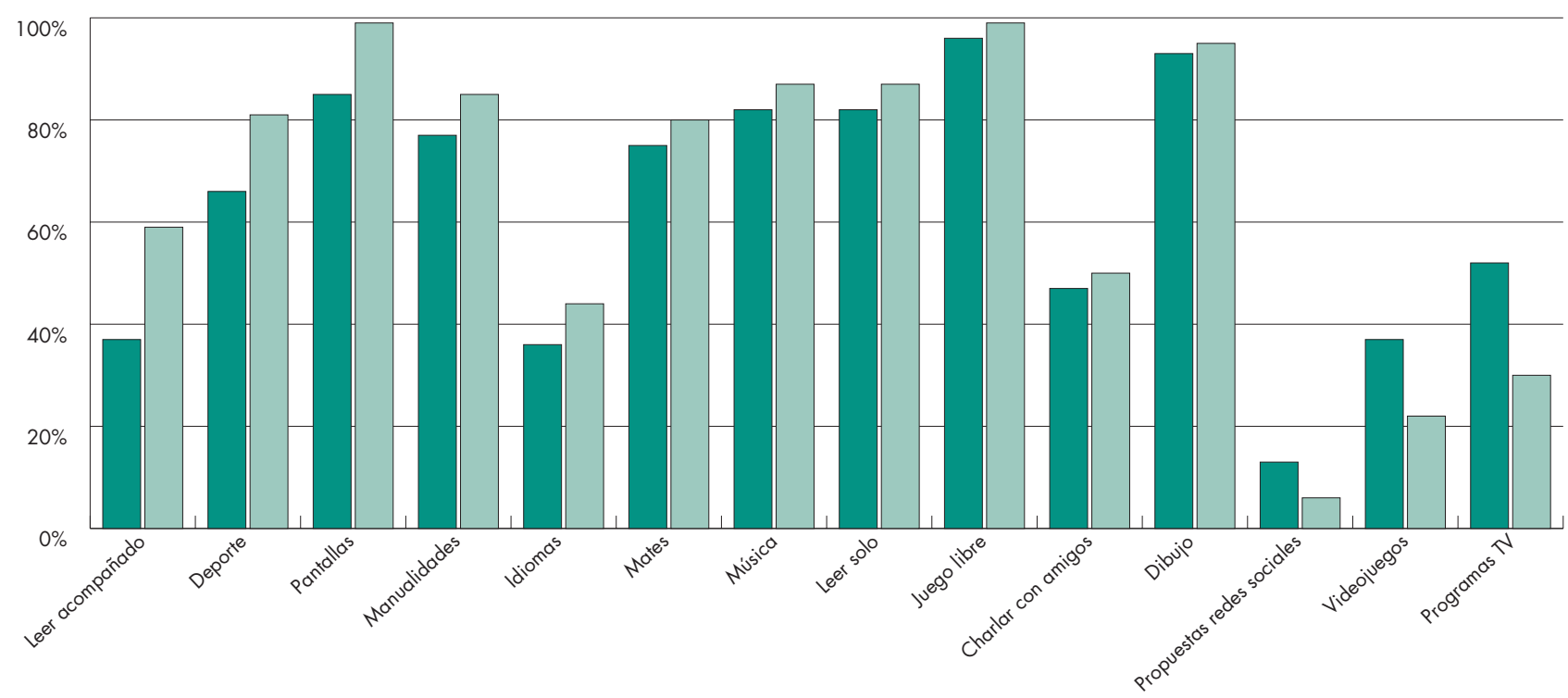

En el caso de los niños mayores, se observan las mismas diferencias, aunque la brecha aumenta en las actividades musicales y otras actividades artísticas y es menos pronunciada en los videojuegos.

En una situación tan extrema, ante la ausencia de la institución escolar, parece que las familias con menos capital cultural hacen un mayor uso de recursos externos para apoyar las actividades de aprendizaje de sus hijos e hijas, mientras que las familias con más capital cultural tienen más confianza en sus habilidades para responder a las necesidades de aprendizaje.

\subsection{Un tiempo «extraescolar» también desigual}

La participación en actividades después de la escuela es una fuente de oportunidades diferenciales de aprendizaje para niños y niñas de diferentes orígenes socioeconómicos (Bradley y Conway, 2016; González Motos, 2016; Lauer et al., 2003; Potter y Morris, 2017). Nuestra encuesta recogió información sobre la participación de los niños y niñas en una o más actividades extraescolares y después del comienzo del confinamiento. La COVID-19 en esta área también han aumentado la brecha entre los y las menores socialmente favorecidos y aquellos desfavorecidos.

Antes del confinamiento, las actividades extraescolares eran más frecuentes durante la educación primaria y particularmente para aquellos niños y niñas cuyas madres tenían un título universitario $(94,2 \%)$ en comparación con aquellos cuyas madres solo tenían educación obligatoria (61\%). Esta 
polarización se observa más acusada particularmente para los niños y niñas que cursan educación secundaria obligatoria ( $81 \%$ frente a $45 \%$ ). El capital cultural y económico de las familias también está asociado con la cantidad de actividades extracurriculares que se realizan. Por ejemplo, para las familias con dos hijos en educación secundaria obligatoria, el número promedio de actividades después de la escuela era de 3,3 actividades en hogares con educación superior y de 1,5 en aquellos con bajo nivel educativo. En conjunto, los deportes, los idiomas extranjeros y las prácticas artísticas son las actividades más frecuentes. Se observan, no obstante, diferencias por nivel de ingresos. Las familias de menor renta (quintil 1) están más representadas en actividades deportivas y en clases de refuerzo escolar, mientras que los hijos e hijas de familias de quintiles más ricos están sobrerrepresentados en extraescolares de idiomas y musicales.

\section{Gráfico 5. Actividades extraescolares antes del confinamiento, según nivel educativo que cursa y nivel instructivo de la madre}

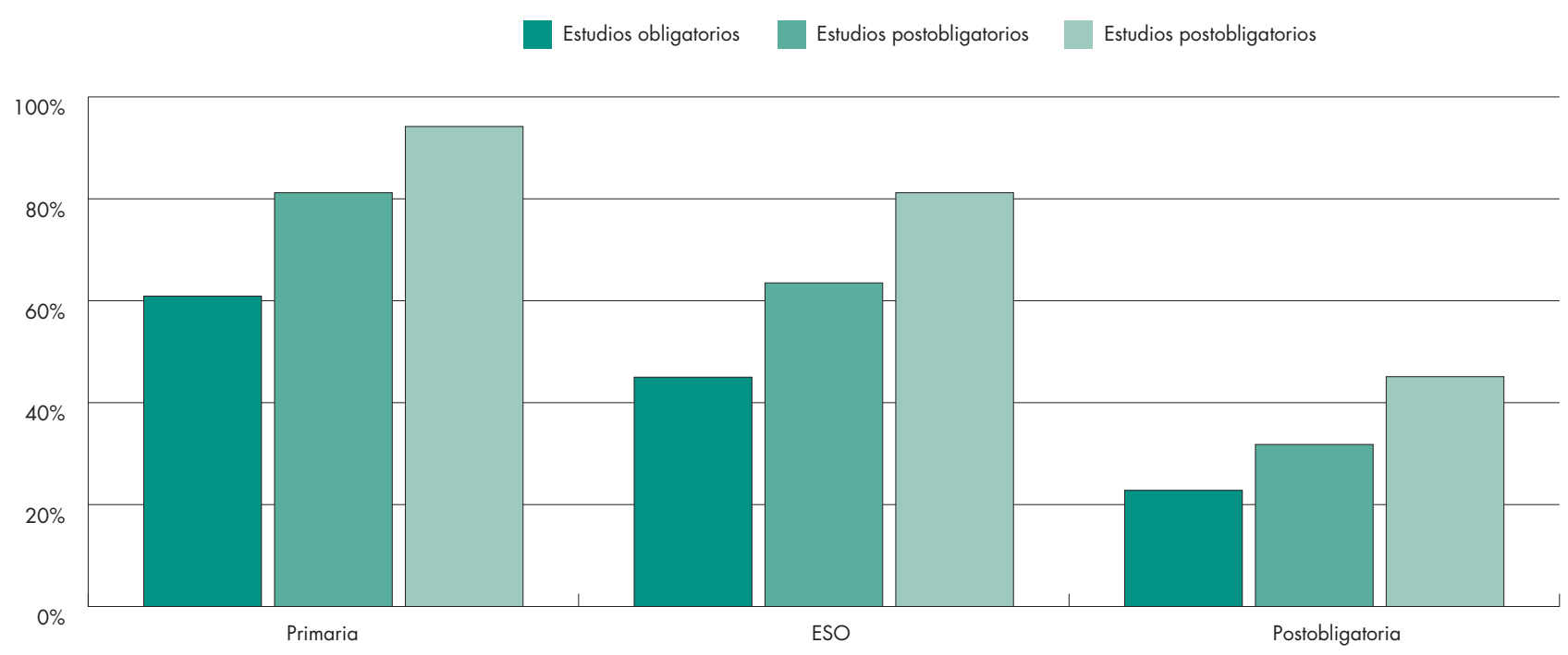

La mayoría de las actividades extraescolares $(70 \%)$ fueron interrumpidas durante el confinamiento. Sin embargo, las desigualdades económicas y culturales también son visibles en la probabilidad de mantener estas actividades durante esta etapa excepcional. El gráfico 6 muestra que los hijos e hijas de familias con un nivel educativo más bajo no solo tenían una menor participación en actividades extraescolares antes del confinamiento, sino que además las mantuvieron en menor medida después del cierre de los colegios. 


\section{Gráfico 6. Continuidad de actividades extraescolares durante el confinamiento, según nivel educativo de la madre}

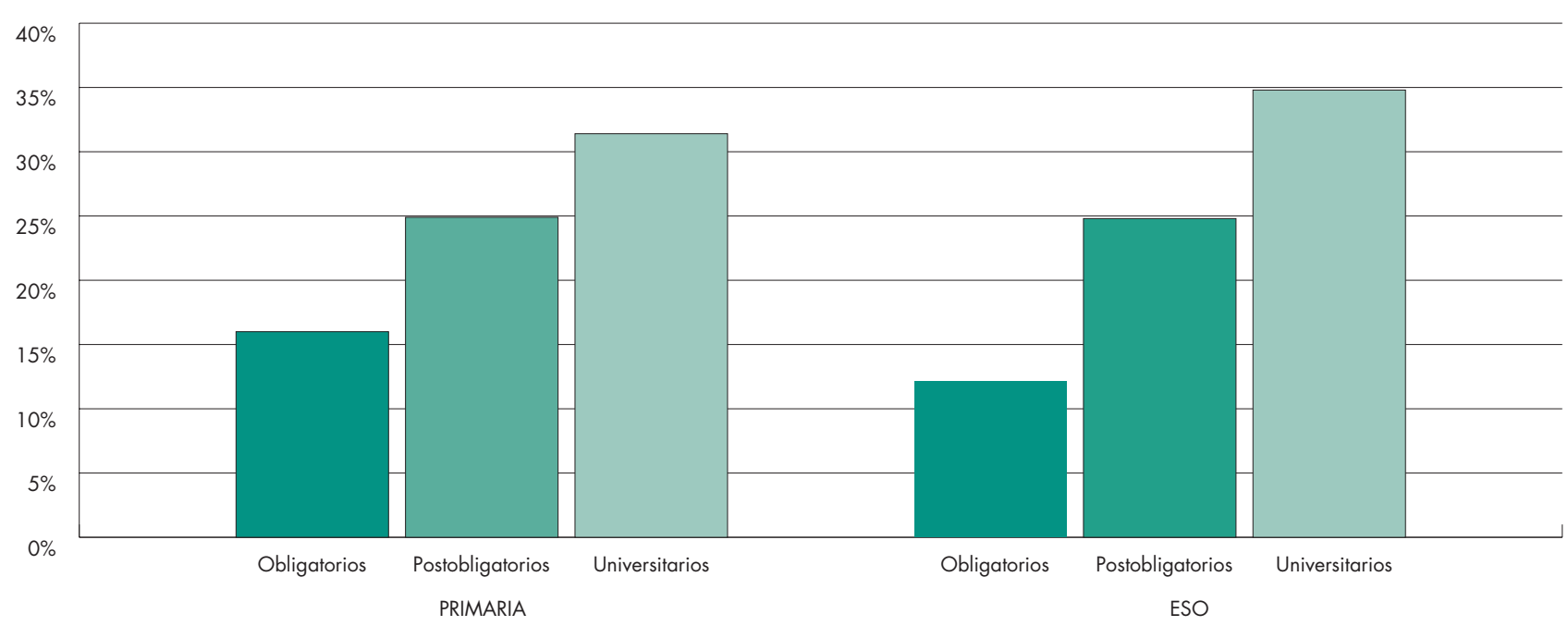

Dos razones permiten entender estas diferencias. En primer lugar, las familias con mayor capital económico y cultural participaban en actividades con mayor probabilidad de continuar a distancia (actividades artísticas, idiomas extranjeros) que las actividades más comúnmente practicadas por familias con menor capital (deportes). En segundo lugar, se observa entre las familias con menor capital cultural y económico un mayor porcentaje de interrupción voluntaria de las actividades extraescolares a pesar de la posibilidad de mantenerlas, probablemente a causa de las dificultades para hacer frente a los costos en tiempos de crisis. A modo de ejemplo, las actividades de educación compensatoria, que podían seguirse en línea, fueron interrumpidas voluntariamente por el $25 \%$ de las familias que las seguían anteriormente. Esta interrupción voluntaria fue particularmente alta entre las familias con educación obligatoria (80\%) en comparación con aquellas con título universitario (62\%).

\section{Conclusiones}

La suspensión de las clases presenciales intentó ser compensada con el establecimiento de actividades a distancia, pero, a pesar de este esfuerzo de reconversión escolar, nuestro análisis refleja desigualdades significativas en la exposición al aprendizaje escolar en función de las características familiares (ingresos, nivel de logro educativo) y características escolares (nivel educativo, titularidad). La brecha digital y la falta de acceso a dispositivos tecnológicos han dejado a algunos niños y jóvenes sin opciones para mantenerse conectados al aprendizaje durante al menos seis meses. E, incluso cuando ha habido conexión, el seguimiento escolar ha sido desigual y la educación a distancia no ha dado respuesta a todas las funciones desarrolladas por la escuela presencial.

Uno de los grandes desafíos post-pandemia será sin duda cómo reducir la desigualdad educativa creciente que está provocando el cierre escolar. La desaparición de la escuela presencial ha extremado el aislamiento y la segregación del alumnado más desfavorecido y sus familias. Nuestro análisis muestra que las familias con un nivel educativo más bajo tienen menos recursos y conocimientos para ayudar a sus hijos con las tareas escolares. Si bien estas desigualdades no son nuevas, tal y como ha puesto de manifiesto ampliamente la literatura, estas limitaciones se han visto agudizadas ante la desaparición de la escuela presencial y sus recursos y el aumento de demanda de trabajo autónomo a los estudiantes. 
Asimismo, el capital cultural y económico familiar también influye en la adopción de prácticas de aprendizaje informal cotidianas que están más o menos alineadas con la lógica escolar, lo que aumenta fácilmente las diferencias en las oportunidades de aprendizaje y la familiaridad con lo que se considera conocimiento válido. Las actividades extracurriculares también se han mantenido más entre los niños y niñas de familias más acomodadas, lo que también ha aumentado las desigualdades previamente existentes.

Los datos apuntan a una clara acumulación de las pérdidas, por lo que los esfuerzos de compensación son ahora aún más necesarios. Hay que pensar en formas intensivas y de aceleración de aprendizaje para el alumnado más desfavorecido y esto debería incluir el tiempo lectivo y el no lectivo. Hay que pensar, pues, en reforzar los mecanismos de acompañamiento al aprendizaje, no sólo con profesorado, sino también con otros profesionales de la atención a las personas y con voluntariado (como ya están haciendo algunos programas de refuerzo escolar).

La pandemia y el confinamiento son una invitación a pensar y repensar varias cuestiones de política educativa, tanto en lo referente a la acción durante un posible nuevo cierre escolar como, especialmente, una vez se pueda volver a la escuela. Los datos disponibles evidencian que efectivamente la brecha digital existe, tanto en acceso como en uso, por lo que las diferentes administraciones hacen bien (aunque llegan tarde) al intentar cerrarla facilitando conexión y dispositivos en los hogares que no tienen.

Sin embargo, esto es insuficiente. Las limitaciones de los sistemas digitales hacen razonable que durante el confinamiento se apueste también por instrumentos alternativos al aprendizaje en línea. Aquí se incluye un mayor uso intensivo de la televisión o la radio como recursos para el aprendizaje, pero también la posibilidad de hacer llegar material escolar y educativo a las familias más desfavorecidas en formatos no digitales.

Se ha evidenciado también la necesidad de mejorar las competencias digitales del alumnado y del profesorado. Hay que incrementar sus competencias, tanto en los usos y conocimientos de la tecnología como, especialmente, en el diseño de una nueva relación enseñanza-aprendizaje en un entorno no presencial. Cómo enseñar es necesariamente diferente, y requiere de habilidades poco trabajadas hasta ahora, tanto por parte del profesorado como del alumnado, pero también hay que redefinir qué se enseña.

Y hay que resolver cuestiones de carácter estructural. Es más necesario que nunca maximizar una composición escolar que beneficie especialmente al alumnado más desfavorecido. Dicho de otro modo, hay que poner esfuerzos para reducir la segregación escolar y facilitar la interacción entre el alumnado que ha gozado de continuidad educativa y el que no. Facilitar espacios escolares y extraescolares para hacerlo posible sería una estrategia innovadora y sin duda eficaz.

\section{Referencias bibliográficas}

Abadzi, Helen (2009): "Instructional Time Loss in Developing Countries: Concepts, Measurement, and Implications”. World Bank Research Observer, 24 (2), 267-290. DOI: https://doi.org/10.1093/wbro/ $1 \mathrm{kp} 008$.

Alegre, Miquel Ángel (2016). Are Summer Programs Effective In Improving Learning And Educational Outcomes In Students? Barcelona: Ivàlua-Fundació Jaume Bofill. http://www.ivalua.cat/documents/1/05_07_2016_10_24_36_Summer_programs_Whatworks_03_.pdf 
Alegre, Miquel Ángel y Benito, Ricard (2012): “Climas y (sobre todo) culturas escolares: cómo se explican y qué permiten explicar” en Carme Gómez-Granell y Pau Marí-Klose (eds.): Familia y relaciones intergeneracionales: un espacio de oportunidades para la educación de los hijos e hijas. Barcelona: Institut d'Infància i Món Urbà.

Blank, Grant (2017): “The Digital Divide Among Twitter Users and its Implications for Social Research”. Social Science Computer Review, 35 (6), 679-697. DOI: https://doi.org/10.1177/0894439316671698.

Bonal, Xavier y González, Sheila (2020): “The Impact of Lockdown on the Learning Gap: Family and School Divisions in Times of Crisis". International Review of Education. DOI: https://doi.org/10.1007/ s11159-020-09860-z.

Borse, Rebekah H.; Behravesh, Casey Barton; Dumanovsky, Tamara; Zucker, Jane R.; Swerdlow, David; Edelson, Paul; Choe-Castillo, Julia y Meltzer, Martin I. (2011): "Closing Schools in Response to the 2009 Pandemic Influenza a H1N1 Virus in New York City”. Economic Impact On Households. Clinical Infectious Diseases, 52 (Suppl_1), 168-172. DOI: https://doi.org/10.1093/cid/ciq033.

Bradley, John L., \& Conway, Paul F. (2016): A Dual Step Transfer Model: Sport and Non-Sport Extracurricular Activities and the Enhancement of Academic Achievement”. British Educational Research Journal, 42 (4), 703-728 DOI: https://doi.org/10.1002/berj.3232.

Burgess, S., \& Henrik Sievertsen, H. (01/04/2020). The impact of COVID-19 on education. VOX, CEPR Policy Portal. Retrieved from https://voxeu.org/article/impact-covid-19-education

Cabrera, Lepoldo (2020): "Efectos del coronavirus en el sistema de enseñanza: aumenta la desigualdad de oportunidades educativas en España”. Revista de Sociología de la Educación-RASE, 13 (2) Especial, COVID-19, 114-139. DOI: http://dx.doi.org/10.7203/RASE.13.2.17125.

Cattaneo, Maria A.; Oggenfuss, Chantal y Wolter, Stefan C. (2017): “The More, the Better? The Impact of Instructional Time on Student Performance”. Education Economics, 25 (5), 433-445. DOI: https:// doi.org/10.1080/09645292.2017.1315055.

Chen, Wan-Chin; Huang, Angela S.; Chuang, Jen-Hsiang; Chiu, Chen-Chung y Kuo, Hsu-Sung (2011): "Social and Economic Impact of School Closure Resulting from Pandemic Influenza A/H1N1". Journal of Infection, 62 (3), 200-203. DOI: https://doi.org/10.1016/j.jinf.2011.01.007.

Coe, Rob; Weidmann, Ben; Coleman, Robbie; Kay, Jon; Ellis-Thompson, Amy; Gnanapragasam, Alexander y Zaman, Mohammad (2020). Impact of School Closures on the Attainment Gap. London: Education Endowment Foundation.

Dahmann, Sarah C. (2015): "How Does Education Improve Cognitive Skills? Instructional Time versus Timing of Instruction”. SOEPpaper, 769. DOI: https://doi.org/10.2139/ssrn.2630179.

Goldstein, Dana (2020). "Research Shows Students Falling Months Behind During Virus Disruptions”. The New York Times (en línea). https://www.nytimes.com/2020/06/05/us/coronavirus-education-lost-learning.html?smid=tw-share. 
González Motos, Sheila (2016). What Impact Do After-School Activities Have on Children's and Youngsters' Learning? What Works in Education? Series, 4. Barcelona: Ivàlua - Fundació Jaume Bofill. https:/ /www.fbofill. cat/sites/default/files/afterschool_Whatworks_04_191016.pdf.

González Motos, Sheila (2020). Are Programmes to Combat School Absenteeism Effective? What works in Education? Series, 17. Barcelona: Ivàlua - Fundació Jaume Bofill (en línea). https://www.fbofill.cat/sites/ default/files/Whatworks_17_070520.pdf, consultado el 8 de septiembre de 2020.

Goodman, Joshua S. (2014): "Flaking Out: Student Absences and Snow Days as Disruptions of Instruction Time. NBER Working Paper Series, Vol. 20221. DOI: https://doi.org/10.3386/w20221.

Gromada, Anna y Shewbridge, Claire (2016): "Student Learning Time: A Literature Review". OECD Education Working Papers, 127. DOI: https://doi.org/10.1787/5jm409kqqkjh-en.

Hanushek, Eric A. (2015): “Time in Education: Introduction”. Economic Journal, 125 (588), F394-F396. DOI: https://doi.org/10.1111/ecoj.12266.

Huebener, Mathias; Kuger, Susanne y Marcus, Jan (2017): “Increased Instruction Hours and the Widening Gap in Student Performance”. Labour Economics, 47, 15-34. DOI: https://doi.org/10.1016/j. labeco.2017.04.007.

Iqbal, Syedah; Azevedo, João; Geven, Koen; Hasan, Amer; Patrinos, Patrinos, Harry A. (2020): "We Should Avoid Flattening the Curve in Education. Possible Scenarios for Learning Loss During the School Lockdowns". Education for Global Development (en línea). https://blogs.worldbank.org/education/we-should-avoid-flattening-curve-education-possible-scenarios-learning-loss-during-school.

Jaume, D. y Willén, A. (2019): The Long-Run Effects of Teacher Strikes: Evidence from Argentina”. Journal of Labor Economics, 37 (4), 1097-1139. DOI: https://doi.org/10.1086/703134.

Kay, Jonathan (2020). Remote Learning. London: Education Endowment Foundation.

Kuhfeld, Megan y Tarasawa, Beth (2020a): "Projecting the Potential Impacts of COVID-19 School Closures on Academic Achievement”. NWEA Research (en línea). https://www.nwea.org/research/publication/projecting-the-potential-impacts-of-covid-19-school-closures-on-academic-achievement/.

Kuhfeld, Megan y Tarasawa, Beth (2020b). “The COVID-19 Slide: What Summer Learning Loss Can Tell us about the Potential Impact of School Closures on Student Academic Achievement". NWEA Research (en línea). https://www.nwea.org/research/publication/the-covid-19-slide-what-summerlearning-loss-can-tell-us-about-the-potential-impact-of-school-closures-on-student-academic-achievement/.

Lauer, Patricia A.; Akiba, Motoko; Wilkerson, Stephanie B.; Apthorp, Helen S.; Snow, David y Martin-Glenn, Mya (2004). The Effectiveness of Out-of-School-Time Strategies in Assisting Low-Achieving Students in Reading and Mathematics: A Research Synthesis (en línea). https://www.researchgate.net/publication/268323547_ The_Effectiveness_of_Out-of-School-Time_Strategies_in_Assisting_Low-Achieving_Students_in_ Reading_and_Mathematics_A_Research_Synthesis.

Marcotte, Dave E. y Hemelt, Steven W. (2008): "Unscheduled School Closings and Student Performance”. Education Finance and Policy, 3 (3), 316-338. DOI: https://doi.org/10.1162/edfp.2008.3.3.316. 
Meyer, Frauke; Meissel, Kane y McNaughton, Stuart (2017): "Patterns of Literacy Learning in German Primary Schools Over the Summer and the Influence of Home Literacy Practices". Journal of Research in Reading, 40 (3), 233-253. DOI: https://doi.org/10.1111/1467-9817.12061.

Mora, Toni y Escardíbul, Josep-Oriol. (2018): “Home Environment and Parental Involvement in Homework During Adolescence in Catalonia (Spain)". Youth and Society, 50 (2), 183-203. DOI: https:// doi.org/10.1177/0044118X15626050.

Oreopoulos, Philip; Page, Marianne E. y Stevens, Ann Huff (2006): “The Intergenerational Effects of Compulsory Schooling”. Journal of Labor Economics, 24 (4), 729-760. DOI: https://doi.org/10.1086/506484.

Pérez, Carmen Nieves; Betancort, Moises; Cabrera, Leoplodo (2013): "Family Influences in Academic Achie-vement. A study of the Canary Islands". Revista Internacional de Sociología (RIS), 71 (1), 169-187. DOI: https://doi.org/10.3989/ris.2011.04.11.

Potter, Daniel y Morris, David S. (2017): "Family and Schooling Experiences in Racial/Ethnic Academic Achievement Gaps: A Cumulative Perspective”. Sociological Perspectives, 60 (1), 132-167. DOI: https:// doi.org/10.1177/0731121416629989.

Rogero-García, Jesús (2020): "La ficción de educar a distancia". Revista de Sociología de la Educación-RASE, 13 (2) Especial, COVID-19, 174-182. DOI: http://dx.doi.org/10.7203/RASE.13.2.17126.

Sadique, M.Z.; Adams, Elisabeth J. y Edmunds, Wiliam J. (2008). "Estimating the Costs of School Closure for Mitigating an Influenza Pandemic”. BMC Public Health, 8, 1-7. DOI: https:/ /doi.org/10.1186/1471 2458-8-135.

Scheerens, Jaap. (2014). Effectiveness of Time Investments in Education. Enschede: Springer.

Shores, Kenneth y Steinberg, Matthew (2018): "The Impact of the Great Recession on Student Achievement: Evidence from Population Data”. CEPA Working Paper, 17-09 (en línea). http://cepa.stanford. edu/wp17-09.

Tarabini, Aina (2020): “¿Para qué sirve la escuela? Reflexiones sociológicas en tiempos de pandemia global”. Revista de Sociología de la Educación-RASE, 13 (2) Especial, COVID-19, 145-155. DOI: https://doi. org/10.7203/RASE.13.2.17135.

Vallespín, Ivanna (2020): "Educació detecta 55000 famílies sense ordinador o sense connexió per poder seguir les classes a distància”. El País (en línea). https://cat.elpais.com/cat/2020/04/08/catalunya/1586359707_283384.html, consultado el 8 de abril de 2020.

Van Lancker, Wim y Parolin, Zachary (2020). “COVID-19, School Closures, and Child Poverty: A Social Crisis in the Making”. The Lancet Public Health, 5 (5): e243-e244. DOI: https://doi.org/10.1016/S24682667(20)30084-0.

Von Hippel, Paul T. (2019): “Is Summer Learning Loss Real?” Education Next, 19 (4), 8-14 (en línea). https://www.educationnext.org/is-summer-learning-loss-real-how-i-lost-faith-education-research-results/. 


\section{Notas biográficas}

Xavier Bonal es Catedrático de Sociología en la Universidad Autónoma de Barcelona, Special Professor of Education and International Development en la Universidad de Amsterdam, director del centro Globalización, Educación y Politicas Sociales (GEPS) y coordinador del Máster Erasmus Mundus GLOBED: Education Policies for Global Development. Ha sido miembro fundador de la red Europea sobre Globalización y Educación (GENIE) y miembro de la red de expertos en ciencias sociales y educación (NESSE) de la Comisión Europea. Ha sido consultor para diversos organismos internacionales, como UNICEF, UNESCO, el Consejo de Europa o la Dirección General de Educación de la Comisión Europea y profesor invitado en diversas universidades europeas y latinoamericanas.

Sheila González es doctora en Políticas Públicas y Transformación Social por la Universidad Autónoma de Barcelona (UAB). Actualmente, es investigadora postdoctoral en el Departamento de Sociología de la UAB. Ha sido investigadora visitante en Hochschule Bremen (Alemania), Instituto de Ciencias Sociales (UNAM, México) y Departamento de Investigaciones Educativas (CINVESTAV-México). Es miembro del Instituto de Gobierno y Políticas Públicas (IGOP) y del centro de investigación Globalización, Educación y Políticas Sociales (GEPS). Sus intereses de investigación incluyen el análisis de políticas públicas y desigualdades sociales, con especial énfasis en la educación y la migración. . 